\title{
An Improved Genetic Algorithm for Training Airspace Planning
}

\author{
Ma Jiacheng ${ }^{1 \mathrm{a}}$, Yao Dengkai ${ }^{1}$, Zhao Guhao ${ }^{1}$ \\ ${ }^{1}$ Air Traffic Control and Navigation College, Air Force Engineering University, Xian, 710000, China \\ aemail: 727032612@qq.com
}

Keywords: airspace planning; genetic algorithm; packing optimization

\begin{abstract}
Airspace planning of tactical training is a centralized planning, which is typical for Air Force tactical training. Because of the complexity of airspace and the diversity of training courses, artificial packing can't guarantee the utilization rate of airspace. Due to the irregularities of airspace, the minimum horizon merit-based insertion algorithm was proposed based on analysis of BL algorithm considering the reasonable utilization of surrounding airspace; On account of airspace limitation, selection operator, crossover operator and fitness function were established based on basic genetic algorithm, and for the purpose of packing optimization, genetic algorithm and improved packing algorithm were combined. The results show that the algorithm can ensure the utilization of airspace. The above method may provide a scientific basis for airspace planning of tactical training in real life.
\end{abstract}

\section{Introduction}

With the advance of the transformation of military training, the proportion of military training time and tactical training has been continuously improved. The joint training of multiple military machines and the training of system confrontation has become the normal situation. Thus, the demand for airspace resources has sharply increased year by year. At the same time, China's civil air transport into a new stage of sustained and rapid development, the demand for airspace is also increasingly urgent. Therefore, the rational planning of tactical training airspace can ease the tension of airspace resourse. The optimizing the airspace structure can effectively alleviate the contradiction between military and civilian airspace requirements.

For the training of airspace planning, there is no relatively mature calculation method and theoretical system at present. It usually rely mainly on staff officers based on human experience design, which also makes the airspace utilization and flexibility are relatively poor. But the assist of computer can improve work efficiency. This kind of problem is similar to the problem of nesting, which cannot be completely solved, because of the high complexity. At present, there are many algorithms to solve this problem, such as tabu search, simulated annealing, genetic algorithms, and so on. The combination the human experience and the heuristic idea to solve the nesting problem of rectangle was provided in Ref.1.The authors packed the bins according to the left-bottom principle. Using GA-based approaches, the two-dimensional bin-packing problem for optimizing the build efficiency was solved in Ref.2.In Ref.3,The authors improved the BL algorithm to better solve the problem of coding transformation. The packing problem of the free-form items was presented in Ref[4].The authors translated irregular pieces into convex polygon. And then the convex polygon is combined into a regular hexagon to solve the problem of arranging the irregular pieces.

Compared with the problem of nesting in Ref. [1-4], the problem of tactical training airspace planning has its own uniqueness. One is the irregularities of the training area. Usually, the airport training area is irregular, which undoubtedly increased the complexity of the problem. However, the genetic algorithm are for the rules of the rectangular area to nest in Ref.[1-3]. The irregular parts packing in the rule area of the nesting problem.is solved in Ref.4. The second is the limited training subjects. the training subjects cannot exceed the specified airspace, otherwise it will bring unnecessary trouble. So not all training subjects can be discharged. This is also the difference between the other nesting problems. The remainder of the paper includes five sections.In Section2, tactical training airspace planning model is described. In Section3, Improved genetic algorithm is 
discussed and a new decoding method is proposed to solve the problem of nesting in irregular regions. In Section4,the process of genetic algorithm is presented. Section 5 includes the results of two application examples and Section 5 concludes the paper.

\section{Tactical Training Airspace Planning Model}

The tactical training airspace planning problem refers to the allocation of multiple training airspace of different sizes in the training area under the jurisdiction of the airport. The goal is to find the largest utilization rate of the administered training area. The principle of the distribution is as follows: (1) The training airspace cannot overlap the others.(2) The airspace occupied by the training subject needs to be allocated within the training area under the jurisdiction of the airport. The whole training area discussed in this paper is convex polygon, and each training subject is abstracted as a rectangular block structure.

The training space of the entire convex polygon can be described by its vertex coordinates in a certain order. The airspace boundary Loop can be represented by Loop $=\{$ P1, P2, P3, P4, P5 $\}$. As shown in Figure 1.
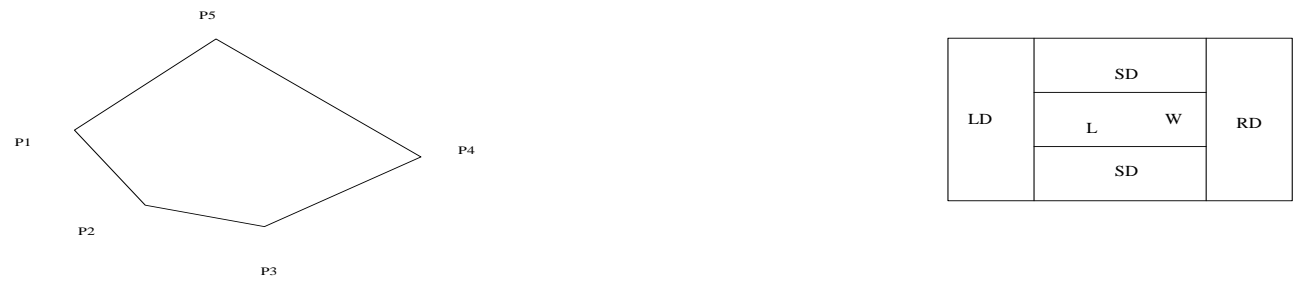

Figure 1 Airspace boundary diagram Figure 2 Schematic diagram of the required airspace for each subject

Each training airspace is a rectangular structure, in addition to the required airspace itself, but also consider the appropriate safety interval. That is, $B=\{L, B, L D, S D, R D\}$, as shown in Fig2.

Training airspace planning is to seek the best- utilization plan according to some certain rules. Each vertex of a rectangle block must not exceed the airspace boundary. As shown in equation 1, the coordinates $\left(\mathrm{X}_{\mathrm{ij}}, \mathrm{Y}_{\mathrm{ij}}\right.$ ) should meet the conditions

$$
\left(X_{i j}, Y_{i j}\right) \in \prod^{(\text {Loop) }}
$$

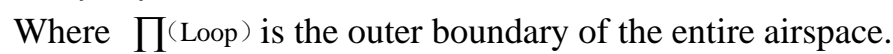

\section{Improved Genetic Algorithm Description}

According to the irregularity, diversity and finiteness of training airspace, this paper firstly preprocesses the whole training area, and then encodes all the tactical training airspace. Based on the BL algorithm, we make corresponding improvements and seek new decoding methods. According to the characteristics of tactical training airspace, the corresponding fitness function is established.

\section{Training Airspace preprocess}

Due to the irregularity of the airspace, the whole training area should be discretized firstly. The concrete steps are as follows:

1) The entire training airspace is stored in the form of images.

2) Convert the image to a bitmap ( $0 / 1$ form) for storage

3) Artificial division of the interval, and take the reliability of 0.75 , the bitmap transform into a matrix V.

Through the above operation, the training area is stored in the form of a matrix for subsequent operations.

\section{Genome Encoding}

The genetic algorithm is considered by the genome, each requiring a different set of specific genetic operators. The genome defines the sequence, $\mathrm{P}$, in which the items are considered for packing. So the final target solution of the problem can be expressed as a string of numbers, ie, $\mathrm{P}=$ 
$\{\mathrm{P} 1, \mathrm{P} 2, \ldots, \mathrm{Pn}\} . \mathrm{n}$ is the general number of the training airspace. $\mathrm{Pi}$ is an integer number $(1 \leqslant|\mathrm{Pi}|$ $\leqslant \mathrm{n}$ ). There are positive and negative points, when the $\mathrm{P}$ is negative when the task that corresponds to the airspace firstly rotated 90 degrees and then participate in emissions ( Default training airspace is wider than high)

The advantage of using this string of numbers is that the permutation scheme can be changed more easily and that each scheme has a unique sequence corresponding to it. Each sequence represents a genome, and subsequent crossover and mutation operator operations operate on the string, resulting in a new solution

\section{Feasible Solution Decoding Method}

After coding the feasible solution, it is the key to find out the corresponding layout chart. It can be further calculated airspace utilization through this layout chart. In [1], the rectangular orthogonal nesting genetic algorithm is studied. The basic idea is to regard the coding of the individual as a permutation, and transform the coding into the corresponding layout chart by BL (Bottom Left) algorithm. But this method is directed against the irregular and limited area for nesting. It is clear that the algorithm is not suited for training airspace planning. Therefore, this paper proposes a new decoding method based on BL algorithm.

For a given problem of irregular training airspace emissions, the strategy adopted in this paper is as follows: For each digit string, search in sequence for the rectangle training space that can be put down until it cannot be allocated within a given irregular train area. Specific steps are as follows:

1) discretize the irregular areas and set the lower boundary of the irregular area as the highest contour line and the lowest horizontal line.

2) For the training airspace $\mathrm{Pi}$, select the lowest horizontal line in the highest contour line as the lowest horizontal line, if there are several segments, select the left section. If the width of the horizontal line is greater than the width of $\mathrm{Pi}$, the area is discharged to the left of the line segment; otherwise, the rectangular area that can be placed is searched backward from Pi.

3) For the training area Pi which has been discharged, it is necessary to check whether the vertex of the training area $\mathrm{Pi}$ is within the given irregular area. If so, this is the final position of the training area. Then, update the highest contour line. Conversely, find the next suitable rectangle training airspace. If none are available, look for a horizontal line that is next to the lowest horizontal line and use it as the new lowest horizontal line.

4) Repeat these two steps until there is no training airspace that can be allocated within the irregular area.

5) According to the final layout matrix to generate layout chart. As shown in Figure 3.
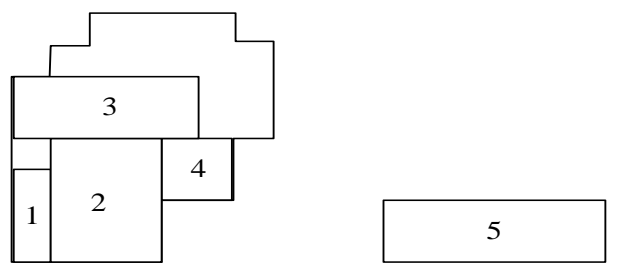

Figure 3 shows a schematic diagram of the decoded emissions

For the discharge of the training area encoded as $\{-1,-2,3,4,5\}$, after the 1st and 2nd airspace is considered, the lowest horizontal line is at the far right side. Because the width of the 3rd training airspace is larger than the width of the lowest horizontal, it cannot be discharged. The 4th area can be discharged and the minimum horizontal line is raised to the upper boundary of the leftmost 1st zone. However, since the width of the remaining training airspace is larger than the horizontal line, So no airspace can be discharged into. Then enhance the 2nd upper boundary as the lowest horizontal line. the 3rd airspace is discharged into the train area and update the horizontal line for the right end of the remaining segments. Since the apex of the 5th training area cannot be completely placed in the area, the decoding process is completed. Finally, in the irregular training area, only 1st, 2nd, 3rd, 4th training airspace can be placed. 


\section{Fitness Function}

In the genetic algorithm, the feasibility of the program is judged through the fitness function. The bigger the fitness value is, the better the program is. In this paper, the compactness of the packing, which is to be maximized, is defined as the packed volume divided by the volume of the whole training area. Due to the finiteness of the training airspace, the greater number of the training airspace which is discharged into is, the better the program is. Therefore, the selection of the fitness function takes into account both the compactness and the number of the training airspace which is discharged into. The fitness function is defined as shown in Eq. (2-3):

$$
\begin{aligned}
& f(p)=n+\text { Aera } / \text { Aera } 0 \\
& \text { Aera }=\sum S_{i}
\end{aligned}
$$

Where $\mathrm{n}$ is the total number of the training airspace which is discharged ; Aera is the total area of the occupied training area; Aera0 is the area below the highest contour line, the highest value of which is 1 . Si is the area of each training airspace.

\section{GA Operators}

Genetic algorithm is a global random search algorithm based on natural selection theory and natural genetic mechanism. Compared with the list method, this method has a unique advantage in solving the problem of global optimization in large space. The genetic algorithm is more convenient to find the most efficient utilization of the planning program.

Initialization of the Population

$\mathrm{N}$ sequences ( $\mathrm{n}$ is an even number) are randomly generated as the initial population, and the fitness of each individual in the population is obtained by using the lower-step algorithm, and it is arranged in ascending order.

\section{Cross Operation}

Individuals in the parent population were randomly paired and crossed. In order to more truly simulate the genetic characteristics of nature, It performs order crossover operation on the orientation part of the genomes. Example: $L 1=\left\{l_{1} 1, l_{1} 2 \ldots l_{1} n\right\}$ and $L 2=\left\{l_{2} 1, l_{2} 2 \ldots l_{2} n\right\}$ are pairs of chromosomes that have been paired. And then randomly selects two numbers $\mathrm{p}, \mathrm{q}(1 \leqslant \mathrm{p} \leqslant \mathrm{q} \leqslant \mathrm{n})$ to carry out the crossover operation. Crossover between the $\mathrm{p}^{\text {th }}$ and the $\mathrm{q}^{\text {th }}$. The specific steps are as follows: $q$ gene is selected as the first $q$ gene of the next chromosome from the $\mathrm{p}^{\text {th }}$ gene on $\mathrm{L} 1$, While the remaining $n-(q-p+1)$ genes are followed in the order they appear on $L_{2}$ to produce a new chromosome. The same operation is done for $L_{2}$ to produce another new chromosome. When $n=6$, $\mathrm{p}=1, \mathrm{q}=4$, as shown in Figure 4:

\begin{tabular}{l}
\begin{tabular}{|l|l|l|l|l|l|l|l|}
\hline $\mathrm{L}_{1}$ & 5 & 6 & 4 & 3 & 2 & 1 \\
\hline $\mathrm{L}_{2}$ & 4 & 3 & 5 & 2 & 1 & 6 \\
\hline
\end{tabular} \\
$\qquad$\begin{tabular}{|l|l|l|l|l|l|l|}
$\mathrm{L}_{1}$ & 5 & 6 & 4 & 3 & 2 & 1 \\
\hline
\end{tabular} \\
\hline
\end{tabular}

\section{Mutation Operation}

At present, there are many common variation methods, this paper uses the method of rotation mutation. The specific operation is as follows: Suppose a small probability Pm, for each gene to generate a random number $\mathrm{P}$, if $\mathrm{P} \leqslant \mathrm{Pm}$ then change its plus-minus. This step means rotated 90 degrees to place the training airspace. There is also a variation for the reverse mutation. It is randomly selected two variation bits. Then it starts to reverse order of the genes between the two variation bits with a certain probability. When the number of genes is relatively small, reverse mutation can improve search efficiency to some degree . But in this genetic algorithm, because in the decoding process has made a dynamic adjustment, the algorithm is only consider the rotation mutation. 


\section{Select Operation}

The n new chromosomes generated by the crossover and mutation operations and their parents' chromosomes are successively solved for their fitness function values, and they are arranged in order of descending fitness value. The first $n$ chromosomes are selected as the parents of the next generation. The above operation is repeated until the value of the fitness function does not change or reaches the predetermined number of iterations to obtain the final optimal solution.

\section{Test results}

In order to test the effectiveness of the genetic algorithm for the problem of training airspace planning, the following examples are presented.

1)The size of different training airspace of the special airport is in Table 1.

Table 1 size of each training area

\begin{tabular}{cccccc}
\hline No. & length & width & No. & length & width \\
\hline 1 & 10 & 8 & 2 & 4 & 4 \\
3 & 5 & 3 & 4 & 6 & 8 \\
5 & 8 & 8 & 6 & 10 & 5 \\
7 & 4 & 14 & 8 & 6 & 6 \\
9 & 7 & 5 & 10 & 4 & 14 \\
11 & 9 & 8 & 12 & 5 & 5 \\
13 & 13 & 10 & 14 & 7 & 8 \\
15 & 6 & 4 & 16 & 7 & 7 \\
\hline
\end{tabular}

It is assumed that the training area under the jurisdiction of the airport is as shown in Fig5

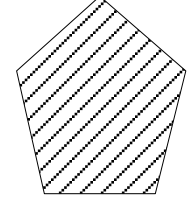

Figure 5: Discrete training airspace

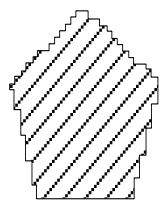

Figure 6 Discrete airspace chart

The irregular region is firstly discretized and a corresponding matrix is generated. The discretization chart is shown in Figure 6.

The genetic algorithm was adopted in training airspace planning. It is assumed that the size of the population is 20 , the number of the iterations is 100 and the probability of mutation is 0.2 . The optimal solution was obtained according to the fitness values. The calculated training airspace planning is shown in Fig7.

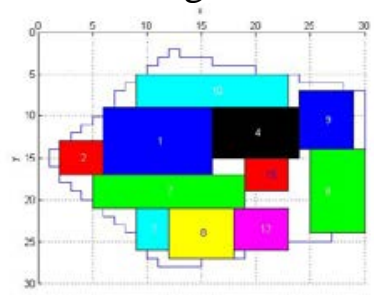

Figure 7 training airspace planning diagram

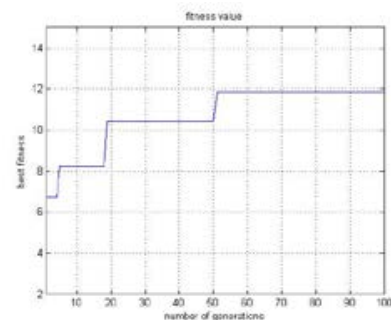

Fig.8 fitness function value

And the optimal solution sequence is 11.8796,In the process of solving, the variation of the fitness value is shown in Figure 8.

2)It is assumed that there are only three types of the training airspaces on the day of the airport, and their sizes are: $(4,14)(8,8)(10,5)$. According to the above method, we can also get the optimal plan, as shown in Figure 9 below; the optimal fitness value is 7.7280 . 


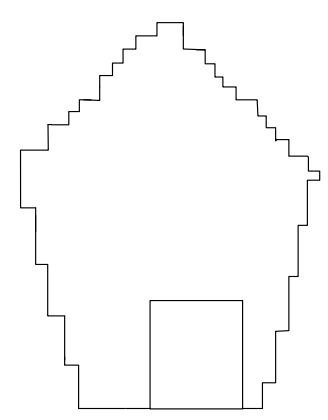

Figure 9 Training airspace planning diagram.

It is shown that this algorithm can solve the problem of training airspace planning. By comparing the utilization of the two instances, it can be found that the algorithm has higher applicability when the training airspace type is larger and the training airspaces is smaller. For the nesting time, compared with the artificial layout, the use of computer to aided nesting greatly shorten the nesting time, especially When the types of training airspace is larger, the effect is more significant.

\section{Conclusion}

In this paper, the problem of training airspace planning is solved by genetic algorithm, and it is transformed into the problem of nesting in finite irregular area. The algorithm is improved on the basis of BL algorithm in the Ref.[1]. Compared with the artificial training airspace planning, the algorithm can improve the efficiency of airspace planning. In this paper, the decoding method and the efficiency and accuracy of the solution need to be improved. These are the next research direction.

\section{Acknowledgement}

In this paper, the research was sponsored by the Key Technique of Unmanned Aerial Vehicle Airspace Safety (KGKT05140501)

\section{References}

[1]YNAASEE H H, ZNIOBER A S. Two-Dimensional Cutting Stock with Multiple Stock Sizes[J]. Journal of the Operational Research Society, 1991, 42(8):672

[2] Jakobs S. On genetic algorithms for the packing of polygons[ $\mathrm{J}$ ] . European Journal Operational Research, 1996, 88,( 1) : 165- 181

[3] Liu Dequan,TengHongfei. On genetic algorithm for the orthogonal packing of rectangles[J].Mini-Micro Systems.1998,19(12):20 25

[4] Zhang Bao, Teng Hangfei, Shi Yanjun. Layout Optimization of Satellite Module Using Soft Computing Techniques. Applied Soft Computing Journal，2008,8(1):507-521.

[5] Andrea L, Silvano M, Michele M. Two-dimensional packing problem: a survey [J] . European Journal of Operation Research, 2002, 141: 241- 252.

[6] Jia Zhixin,YinGuofu,LuoYang,et al.Application of simulated annealing to the rectangular packing problem[J].Journal of Sichuan University,2001,33(5):35 38

[7] Liu De- quan, Teng Hong- fei.An improved BL- algorithm for geneticalgorithm of theorthogonal packing of rectangles[J].European Journalof Operational Research, 1999, 112: 413420.

[8] Liu Jimi, Zhang Shengnan, Huang Youqun. Research and implementation of two dimensional irregular auto-nesting algorithm[J]. Journal of Computer-Aided Design \&Computer Graphics,2000,12(7):488〜491 